\title{
Orthopaedic surgery for carpal tunnel syndrome
}

\author{
Georgi P Georgiev ${ }^{*}$, Vesselin Karabinov ${ }^{2}$ and Martin Apostolov ${ }^{3}$ \\ ${ }^{1}$ Department of Orthopedics and Traumatology, University Hospital Queen Giovanna-ISUL, Medical University of Sofia, Bulgaria \\ ${ }^{2}$ Clinic of Neurology, National Heart Hospital, Sofia, Bulgaria \\ ${ }^{3}$ Student at Medical University of Sofia, Sofia, Bulgaria
}

\begin{abstract}
Carpal tunnel syndrome is the most commonly occurring mononeuropathy, affecting between $2 \%$ and $5 \%$ of the population and is more commonly present in women. Patients may experience a wide range of symptoms, the most frequent of which is a change in sensation or pain in the area of the arm, wrist, or forearm. The pathology is associated with the compression, clamping, or irritation of median nerve in the carpal tunnel. Diagnosis can be based on a medical examination, supported by electrophysiological and ultrasound results. Although spontaneous improvement is possible, in general symptoms worsen. Therefore, early recognition of carpal tunnel syndrome, through clinical and instrumental investigations, is essential for preventing disturbance of hand functions and permanent nerve damage. In the early stages of the disease, treatment is mostly conservative. In case conservative treatment fails, and in medium to severe forms of the disease, surgical intervention is performed. In the present paper we review the literature on the various techniques of operative treatment - open surgery, endoscopic and mini-invasive percutaneous technique under echographic control.
\end{abstract}

\section{Introduction}

Carpal tunnel syndrome (CTS) is the most commonly occurring compressive mononeuropathy of the upper limb, affecting more than $5 \%$ of the general population and $6 \%$ of women over the age of 40 years [1]. The disease is about five times more commonly found in women. CTS may occur at any age, but most commonly affects patients aged between 40 and 65 years and those over 80 years of age [1,2]. CTS may be unilateral or affect both hands, although the bilateral manifestation is much more common: in $22 \%-87 \%$ of patients, in most studies $60 \%$ of all cases [1]. CTS occurs in $14 \%$ of diabetic patients without polyneuropathy and in those with diabetic polyneuropathy the incidence increases to $30 \%$ [3]. In pregnant women, the incidence varies from 7 to $62 \%$, with complaints disappearing in most cases after birth [4].

CTS is the most common musculoskeletal disorder in most European countries, the United States and Canada. In the United Kingdom, the incidence of CTS is between 7 and $16 \%$, which is much higher than in the United States - 5\% [5]. In all Western European countries there has been an increase in work-related musculoskeletal disorders caused by overwork and recurrent unilateral movements. In Europe, more than $60 \%$ of the work-related musculoskeletal disorders of the upper limb are caused by CTS [6].

Surgical treatment is used in patients in whom conservative treatment is ineffective for pain relief, or with progressive motor deficits, severe sensory deficits, or severe electrophysiological abnormalities. Surgical treatment usually leads to the disappearance of the symptoms, or at least prevents subsequent clinical deterioration, particularly the development of motor deficiency. The first decompression of the median nerve $(\mathrm{MN})$ was carried out by Herbert Galloway in 1924. Today surgical treatment of CTS is one of the most commonly performed surgical interventions in France, with around 140,000 cases reported annually. After 1990 there has been a significant increase in interventions. According to data from the French Agency for Hospital
Information (ATIH), the number of cases has increased from 9537 in 1995 to 142,405 in 2005, with their number remaining constant in the coming years. By comparison, 576924 such procedures were performed in the United States in 2006 [3].

Surgical treatment of CTS consists in the decompression of the carpal tunnel by the opening of the flexor retinaculum (retinaculotomy) by open or endoscopic surgical technique. The operation is usually performed under local anesthesia and with the application of a pneumatic tourniquet on the arm. In some cases, retinaculotomy is also associated with neurolysis, epineurotomy or synnectomy $[7,8]$.

The aim of this paper is to review the literature on surgical techniques such as classical open decompression, endoscopic technique and new trends in surgical treatment of CTS, used by hand surgeons.

\section{Open (classic) surgical technique}

This procedure uses a palmar longitudinal incision of about $3-4 \mathrm{~cm}$ in line with the fourth finger, without passing through the distal edge on the Kaplan line or the thenar crease, from the distal transversal skin fold of the wrist to the middle of the palm. The open operation requires an access of 4-5 cm. A mini-incision access has been described, where the required incision is $2 \mathrm{~cm}$. Subcutaneous dissection is required in order to protect the superficial sensory branches, the opening of the flexor retinaculum (FR) is done using a scalpel. In $15 \%$ of people, a sensory branch at the distal end of the incision may intersect the operative field.

*Correspondence to: Georgi P Georgiev, MD, PhD, Department of Orthopaedics and Traumatology, University Hospital Queen Giovanna - ISUL, Medical University - Sofia 8 Bialo more str. BG 1527 Sofia, Bulgaria, Tel: +359884 493523; E-mail: georgievgp@yahoo.com

Key words: carpal tunnel, review, surgical treatment

Received: October 02, 2018; Accepted: October 12, 2018; Published: October 17,2018 
Also the possibility of a transligamental passage of the motor branch for the muscles of the thenar must be verified. The superficial palmar artery at the distal and ulnar section of the incision must be located and protected. Under the protection of a mosquito clamp, the FR is slowly and gradually cut in a distal to proximal direction in the most ulnar part of FR [7].

$\mathrm{MN}$ is inspected. If the epineurium is fibrous, it is carefully cut along the constriction. Epineurotomy is performed under magnification. The cuff is relaxed and a hemostasis is performed. The skin stitches are made in one plane. Synnectomy of flexor tendons is not indicated, except in the case of reactive synovitis and in hemodialysis patients [7].

In a Bulgarian retrospective study of 292 patients with CTS, on the sixth month $83 \%$ of the patients had fully recovered, $13 \%$ had a good level of recovery and $4 \%$ had not recovered the functionality of their arm. Patients who did not achieve complete recovery had diabetes mellitus. An open operation was performed in all patients [9].

\section{Endoscopic technique}

Endoscopic surgery was first demonstrated in the 1990s and is considered to be a safe technique that allows the reduction of morbidity and postoperative recovery time.

Endoscopic techniques have been introduced to reduce the length of the incision and thus potentially reduce post-operative discomfort. Several endoscopic systems have been described, but the risk of complications, including iatrogenic nerve damage, poor visualization, inability to identify anatomical variations, incomplete relief and lesser costs associated with this treatment are still being discussed. Supporters of endoscopic technique recommend courses on cadavers before performing it in clinical practice.

Several techniques have been described for the treatment of CTS, however only two have been validated in practice: the Agee's singleport technique and Chow's two-port technique.

\section{Single-port technique}

Agee et al. [10] describe this technique for the first time. Under a tourniquet, with regional or local anesthesia, and a transversal access of 2 to $3 \mathrm{~cm}$. in the area of the distal groove of the joint between the tendons of flexor carpi radialis and flexor carpi ulnaris muscles. A U-shaped flap is formed on the distal-base of the forearm fascia. Using probes of different diameters, a tunnel is formed for a special endoscopic probe with visual control and a blade for retinaculotomy, which is dispensed distally from the ulnar side of the carpal tunnel radially by hamulus ossis hamati. The wrist is placed in a slightly extended position, and the probe with the blade is inserted into the inner edge of the FR, while the tip is palpated with the other hand. After localization of the distal edge of the FR, the blade opens and the FR is cut from distal to proximal, then re-visualized the cut retinaculum. Agee et al. [10] recommend in case of poor visibility to switch to an open operation.

\section{Two-port endoscopic technique}

Chow [11] describes this technique for the first time. It is performed with local anesthesia and preferably by surgeon - with an esmarch. A marker shows the accesses. Initially, the first line is drawn by passing a line 1 to $1.5 \mathrm{~cm}$ radially from the proximal pillar of os pisiforme. A second line is drawn $0.5 \mathrm{~cm}$ proximal to the first line. From the end of the second line, a third line is made radially, about $1 \mathrm{~cm}$ in size. This line, namely, includes one access.
Several lines are used to locate distal access. The first one starts from the distal end of the fully abducted thumb, transversely to the palmar arm, and a vertical line in the third intercarpal space. At $1 \mathrm{~cm}$, proximal to the intersection of the two lines, a distance of about $0.5 \mathrm{~cm}$ is made. Through proximal access, in depth, it penetrates through a longitudinal section of the fascia and locates the proximal end of FR. A curved cannula dissector is passed through the forearm axis to release synovial tissue from the lower surface of the FR. The hand is lifted from the table, the wrists and fingers being hyperextensive. The assembled cannula is moved in a distal direction, the tip being palpated at the exit portal in the palm. The endoscope is inserted proximal and moved distally to identify the distal end of the FR. The blade in the probe is used to release the distal edge of FR in distal to proximal direction. A triangular blade is used to release the middle portion of the FR, and then a retrograde blade is positioned through the distal access and is drawn distally to join the first cut, the aim being to release the entire distal portion of the FR. The endoscope is drawn from proximal and is moved through the distal access, and the probe blade is used to release the proximal end of the FR. The retrograde blade is placed in the middle of the FR and is drawn proximally until its full release. The forearm fascia is released through the proximal incision with scissors and then the skin is sutured [12].

In the long term, efficacy in terms of recovery after six months and reduction of complaints is the same for both techniques. Studies comparing endoscopic to classic open surgery show that the smaller the access is, the fewer the complications are and the faster the recovery. Hamed et al. [13] in a study of 40 patients, compared the technique by two incisions (limited palmar and mini-incinsion at the level of the distal armpit of the forearm) with conventional open surgery. The authors conclude that there is a significant reduction in postoperative pain and scar sensitivity in favour of the mini-invasive technique.

\section{Ultrasound guided minimally invasive technique}

In recent years, imaging, and in particular ultrasound, has played an increasing role in the diagnosis and treatment of CTS $[2,14]$. Following the establishment of ultrasound criteria for reliable diagnosis of CTS and the possibility it gives for the assessment of the structure of the carpal tunnel and the possible presence of formations or variations, the use of ultrasound imaging in the treatment of CTS has become a fully justified option [15-19].

The most important reason for the development of this new technique is its minimally invasive nature. The open operation requires an access of 4-5 cm, the mini-incision - of $2 \mathrm{~cm}$, the endoscopic of 1-2 $\mathrm{cm}$, while under ultrasound control the access is up to 10 times smaller - in the range of $0.1-0.5 \mathrm{~cm}$.

In 1997, Nakamichi [20] proposed the use of ultrasound imaging during the classical open surgery. Over time, several teams have been able to prove that it is possible to perform the operation only under ultrasound guidance $[3,19]$.

Petrover et al. [21] presented for the first time a minimally invasive ultrasound-guided technique. The patient's arm is placed in a supinated position on the operating table, without a tourniquet, under strictly aseptic conditions and local anaesthesia. Needles 25G and 21G, scalpel №15, and a hook-like knife with a retrograde blade are required. Initially, through echography, the safety zone between the ulnar artery and the MN, the superficial palmar arc, as well as variations of the motor branch were localized. Numerous ulnar nerve variations, as well as the possible passage of the MN motor branch through the FR, are the 
most important risk factors for iatrogenic lesions [22,23]. Initially, local anesthesia is applied to the proximal groove of the wrist, while a second deeper infiltration with Xylocaine $1 \%$ allows for better hydro dissection of the carpal tunnel. Through a pinch-incision with the tip of the scalpel, the hook-like blade is inserted, under ultrasound control under the FR until it reaches a few millimetres distally from the FR, with care being taken to avoid the $\mathrm{MN}$, the beginning of its sensory branches, and the Berrettini branch. The hook is then turned vertically and the FR is cut in a retrograde direction under ultrasound control. Operating time averages $5.8+/-2.4$ minutes, depending on the anatomy and the time required for adequate anesthesia. A post-operative compressive bandage is recommended, as well as rest and, if necessary, non-opioid analgetics [21].

The bandage is removed on the third day and the postoperative clinical examination on the sixth day shows a complete healing of the millimetre incision in all patients. Petrover et al. have not observed any complications [21].

\section{Conclusion}

In case conservative treatment for CTS fails, surgical decompression of the $\mathrm{MN}$ is recommended. Numerous techniques for decompression have been described, ranging from open classic surgery to various mini-incision techniques, endoscopic decompression to minimallyinvasive percutaneous ultrasound-guided technique. A comparison of the surgical procedures shows that the smaller the access, the faster the patient returns to the workplace, but the rate of iatrogenic lesions is greater. Although the release of the $\mathrm{MN}$ is the goal of the treatment, possible injury to even a few nerve fascicles may have adverse effects on the patient. The surgeon should choose the most appropriate surgical method which offers the best visualization of the nerve in order to avoid further damage, taking into account the operator's knowledge and experience as well as the specifics of each individual patient.

\section{Author Contributions}

All authors conceptualized the study and participated in study screening, selection, data extraction and manuscript preparation. All authors provided intellectual content and approved the manuscript for publication.

\section{Conflict of Interest Statement}

No conflicts declared.

\section{Funding}

The authors have nothing to disclose.

\section{References}

1. Dec P, Zyluk A (2018) Bilateral carpal tunnel syndrome - A review. Neurol Neurochir Pol 52: 79-83. [Crossref]

2. Karabinov V, Georgiev GP (2017) Carpal tunnel syndrome - frequent and underestimated condition. MD Journal 4: 75-78. [In Bulgarian]
3. Petrover D, Richette P (2017) Treatment of carpal tunnel syndrome: from ultrasonography to ultrasound guided carpal tunnel release. Joint Bone Spine [Crossref]

4. Osterman M, Ilyas AM, Matzon JL (2012) Carpal tunnel syndrome in pregnancy. Orthop Clin North Am 43: 515-520. [Crossref]

5. Aroori S, Spence RA (2008) Carpal tunnel syndrome. Ulster Med J77: 6-17. [Crossref]

6. Ibrahim I, Khan WS, Goddard N, Smitham P (2012) Carpal tunnel syndrome: a review of the recent literature. Open Orthop J 6: 69-76. [Crossref]

7. Matev I (1983) Decompression of n. medianus in carpal canal. In: Boychev`s operative orthopedics and traumatology. Vol 1. Sofia. Med and Fis. p264.

8. Matev B (2001) Operative treatment of carpal tunnel syndrome - modern trends. Bulg J Orthop Traumatol 36: 550-551.

9. Georgiev GP, Karabinov V, Matev B, Iliev A, Kotov G, et al. (2017) Carpal tunnel syndrome treatment with open surgical release: a study in 292 patients. Acta Morphol Anthropol 24: 76-81.

10. Agee JM, McCarroll HR Jr, Tortosa RD, Berry DA, Szabo RM, et al. (1992) Endoscopic release of the carpal tunnel: a randomized prospective multicenter study. $J$ Hand Surg Am 17: 987-995. [Crossref]

11. Chow JC (1994) Endoscopic carpal tunnel release. Two-portal technique. Hand Clin 10: 637-646. [Crossref]

12. Szabo R (1998) Entrapment and compression neuropathies. In: Green DP, Pedersen W, Hotchkiss RN, eds. Operative Hand Surgery, (4thedn), St. Louis: Churchhill Livingstone. 1417-1446.

13. Hamed AR, Makki D, Chari R, Packer G (2009) Double - versus single-incision technique for open carpal tunnel release. Orthopedics 32: 10. [Crossref]

14. Georgiev G, Karabinov V, Apostolov M, Grudeva V (2018) The role of echography for evaluation of carpal tunnel. Roentg Radiol 57: 197-201. [In Bulgarian]

15. Georgiev GP, Jelev L, Stokov L (2011) Anomalous muscle of the wrist. Case report Health and Sci 1: 17-18. [In Bulgarian]

16. Georgiev GP, Jelev L, Surchev L (2008) Reversed palmaris longus muscle simulating soft-tissue mass as a possible cause of median nerve compression. Bulg J Orthop Traumatol 45: 92-94.

17. Fried SM, Nazarian LN (2018) Ultrasound-guided hydroneurolysis of the median nerve for recurrent carpal tunnel syndrome. Hand. [Crossref]

18. Gruber L, van Holsbeeck MT, Khoury V, Deml C, Gabl MF, et al. (2018) Compliance assessment and flip-angle measurement of the median nerve: sonographic tools for carpal tunnel syndrome assessment? Eur Radiol. [Crossref]

19. 19. Guo XY, Xiong MX, Lu M, Cheng XQ, Wu YY, et al. (2018) Ultrasound-guided needle release of the transverse carpal ligament with and without corticosteroid injection for the treatment of carpal tunnel syndrome. J Orthop Surg Res 13: 69. [Crossref]

20. Nakamichi K, Tachibana S (1997) Ultrasonographically assisted carpal tunnel release. $J$ Hand Surg Am 22: 853-862. [Crossref]

21. Petrover D, Silvera J, De Baere T, Vigan M, Hakime A (2017) Percutaneous ultrasound guided carpal tunnel release - study upon clinical efficacy and safety. Cardiovasc Intervent Radiol 40: 568-575. [Crossref]

22. Petrover D, Bellity J, Vigan M, Nizard R, Hakime A (2017) Ultrasound imaging of the thenar motor branch of the median nerve: a cadaveric study. Eur Radiol 27: 4883-4888. [Crossref]

23. Smith J, Barnes DE, Barnes KJ, Strakowski JA, Lachman N, et al. (2017) Sonographic visualization of thenar motor branch of the median nerve: a cadaveric validation study. PMR 9: 159-169. [Crossref]

Copyright: (C2018 Georgiev GP. This is an open-access article distributed under the terms of the Creative Commons Attribution License, which permits unrestricted use, distribution, and reproduction in any medium, provided the original author and source are credited. 